Recepción: 17 / 10 / 2016

Aceptación: 20 / 12 / 2016

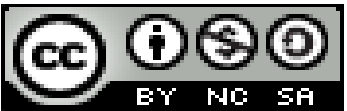

Ciencias económicas y empresariales

Publicación: 15 / 02 / 2017

Artículo de investigation

\title{
Características del neuromarketing como herramienta de posicionamiento empresarial
}

Characteristics of neuromarketing as a tool for business positioning

Características neuromarketing como uma ferramenta para o posicionamiento corporativo

\author{
Carlos E. Rivas-Vallejo ${ }^{\mathrm{I}}$ \\ carloserivas@gmail.com \\ Mariuxi A. Guijarro Cagua II \\ mariuxiguijarro@gmail.com
}

Correspondencia: carloserivas@gmail.com

\footnotetext{
${ }^{\text {I } M a g i s t e r ~ e n ~ A d m i n i s t r a c i o ́ n ~ d e ~ E m p r e s a s, ~ I n g e n i e r o ~ C o m e r c i a l, ~ D o c e n t e ~ d e ~ l a ~ U n i v e r s i d a d ~ T e ́ c n i c a ~ L u i s ~}$ Vargas Torres, Esmeraldas, Ecuador.

II Magister en Administración de Empresas, Ingeniero en Administración Pública, Tecnólogo en Administración Pública, Docente de la Universidad Técnica Luis Vargas Torres, Esmeraldas, Ecuador.
} 


\section{Resumen}

La selección del tipo de estrategia de mercado de una empresa, demanda del conocimiento de las necesidades e intereses de los usuarios. En la actualidad, ciertas empresas se proponen investigar ciento por ciento la forma de pensar de sus clientes. Los avances en la neurociencia y el conocimiento de las reacciones del cerebro ante los estímulos externos se emplean en el campo del marketing en aras de lograr la mayor eficacia posible. El presente texto profundizó en las características más significativas del neuromarketing como herramienta para contribuir al posicionamiento empresarial.

Palabras clave: neuromarketing; neurociencia; estrategia de mercado.

\section{Abstract}

The selection of the type of market strategy of a company, demand of the knowledge of the needs and interests of the users. At present, certain companies intend to investigate one hundred percent the way of thinking of their customers. Advances in neuroscience and knowledge of the reactions of the brain to external stimuli are used in the field of marketing in order to achieve maximum efficiency. The present text delves into the most significant features of neuromarketing as a tool to contribute to business positioning.

Keywords: neuromarketing; neuroscience; market strategy.

\section{Resumo}

A seleção do tipo de estratégia de mercado de uma empresa, exigem o conhecimento das necessidades e interesses dos usuários. Atualmente, algumas empresas planejam investigar cem por cento a maneira de pensar de seus clientes. Os avanços na neurociência e conhecimento das reações do cérebro a estímulos externos utilizados na área de marketing, a fim de alcançar a maior eficiência possível. Este trabalho investigou as características mais significativas do neuromarketing como uma ferramenta para ajudar posicionamento corporativo.

Palavras chave: neuromarketing; neurociência; estratégia de marketing.

\section{Introducción}

El diseño de las estrategias de marketing es uno de los principales aspectos a trabajar dentro del marketing. Las estrategias de marketing definen como se van a conseguir los objetivos comerciales de una empresa. Para ello, es necesario identificar y priorizar aquellos productos que 
tengan un mayor potencial y rentabilidad, seleccionar al público al que se va a dirigir, definir el posicionamiento de marca que se quiere conseguir en la mente de los clientes, y trabajar de forma estratégica las diferentes variables que forman el marketing mix.

La determinación del tipo de estrategia de mercado que se va a ejecutar en una empresa, requiere del dominio de las necesidades e intereses de los usuarios presentes y potenciales, en aras de que se pueda responder en todo momento a lo que el cliente espera de la empresa. Esto ha incidido en que a través del tiempo, el marketing haya ido evolucionando, lo que ha llevado a que en la actualidad existan empresas que se propongan investigar ciento por ciento la forma de pensar de las personas, sea este a nivel cuantitativo o cualitativo, cuyos resultados determinarán los tipos de estrategias a aplicar.

Las campañas han obtenido resultados variables de sus procesos y razonan en base al éxito y no en base a la campaña elaborada o resultado obtenido en un determinado tiempo, por lo que en los últimos años se ha determinado al cliente como parte fundamental dentro de proceso al adquirir o consumir un producto, bien o servicio, llegando a realizar estudios enfocados directamente a ellos con el propósito de ver que tan cambiante puede ser al momento de hacer una elección.

La aplicación de estrategias que sirvan para conocer el comportamiento se llama Neuromarketing, y tiene como propósito decodificar procesos que se dan en la mente del consumidor, con el fin de descubrir las necesidades y poder ofrecer lo que requieran para satisfacerlas. Esto es posible gracias a la tecnología en imágenes de neurociencia que ha forjado una relación estrecha entre empresas y sus clientes.

El presente artículo se propone profundizar en las características esenciales del neuromarketing y su importancia como herramienta esencial para lograr el posicionamiento empresarial.

\section{Desarrollo}

El marketing, es el arte de comercializar productos y servicios de manera rentable para la empresa, a través de la satisfacción de los clientes. Estos poseen necesidades muy diferentes, mientras algunos buscan el precio como prioridad para la compra, otros buscan la calidad del producto, o el prestigio que este concede. Por lo tanto, las mismas empresas, que comercializan los mismos productos buscan maneras diferentes de comercialización. 
El desarrollo del marketing en una empresa persigue los siguientes objetivos:

- Aumento de la cifra de negocios y de los beneficios

- Crecimiento de la cuota de mercado

- Mejorar el posicionamiento del producto

- Penetración en un nuevo mercado

- Aumento de la satisfacción del cliente

En la mercadotecnia, se ha incorporado en los últimos años nuevas tendencias para determinar el comportamiento del consumidor, es así que, al incorporar a los clientes como factor transcendental en el proceso de compra, las empresas buscan conocer más a sus clientes, por ello la tendencia en la utilización de neurociencias aplicadas al marketing.

Los avances en la neurociencia y el conocimiento de las reacciones del cerebro ante los estímulos externos están fuera de toda duda, y sus aplicaciones en el campo del marketing son una de las claves que más expectativas abren para lograr la eficacia de las acciones de marketing.

La neurociencia ha confirmado algo que ya era conocido: lo que dicen y piensan las personas no siempre coincide, y menos aun lo que dicen que van a hacer y luego hacen, o lo que dicen sentir y sienten en realidad. Igualmente, es sabido que la gran mayoría de las decisiones de compra se toman por procesos más emocionales que racionales o, en todo caso, los consumidores racionalizan decisiones que ha tomado antes de forma emocional. Las estrategias ganadoras en campañas de marketing son las que mezclan lo racional y lo emocional, y en las marcas más asentadas, la estrategia emocional pura tiene mejor resultado que la solo racional.

Esto da origen al Neuromarketing, por la creciente necesidad de las empresas de conocer los intereses de los consumidores con el fin de satisfacerlos adecuadamente, por lo que el marketing acudió a la neurociencia para determinar las sensaciones que experimenta el consumidor (Cayuela 2012).

En la actualidad, el Neuromarketing es un factor común en las empresas que buscan efectividad, a través de la actividad cerebral o experiencia del consumidor durante un proceso o adquisición de algún producto, bien o servicio, lo que permite inquirir que niveles de atención está mostrando 
el consumidor, llegando así a averiguar, conocer o entender el estado emocional de una persona cuando un producto aparece en pantalla.

El mundo de los negocios se ve influenciado por gustos y preferencias del consumidor; estudiar cada uno de sus componentes demanda especializarse en el campo, por ende, el ser humano es complicado en el momento de hacer una elección, por lo que se requiere analizar, observar y estudiar cada uno de los movimientos y expresiones para determinar posibles factores que inciden en el momento de adquirir un producto, bien o servicio.

Por eso el Neuromarketing es utilizado como una herramienta, que sirve para el análisis del comportamiento del consumidor enfocado a diferentes áreas como, la recordación de marca, tratando de conocer el pensamiento del consumidor, teniendo una perspectiva más allá de lo que expresa o es capaz de expresar al momento de analizar al consumidor, ya que hay que saber reconocer que aunque se crea que se conoce todo lo que piensa realmente el consumidor, no es tan fácil deducirlo.

Según Misiego (2012), "el Neuromarketing puede definirse como una disciplina avanzada que investiga y estudia los procesos cerebrales que explican la conducta y la toma de decisiones de las personas en los campos de acción del marketing tradicional".

El Neuromarketing se enfoca a procesos cerebrales, la elección por emoción para la toma de decisiones dependiendo de la atención de nuestro entorno con el fin de poder determinar o predecir su conducta frente a una situación de consumo o compra, por lo que llama la atención de la comunidad empresarial tratando de ver la importancia, comprender las emociones y de medirlas.

El Neuromarketing se ve en las reacciones del consumidor de acuerdo a un ambiente donde se desenvuelve al momento de la adquisición de un bien, producto o servicio, como, por ejemplo: imágenes, anuncios, música, sabor, olor, color e intercede en la toma de decisiones y que puede afectar un mercado por la percepción que se crea al momento de decidir.

La aplicación del Neuromarketing como alternativa, se puede utilizar en múltiples áreas del marketing, como, por ejemplo:

- Investigación de mercados 
- Investigación de comportamiento de clientes

- Validación de comerciales de televisión

- Diseño de productos

- Impacto de publicidad

Otro de los autores que se refiere al neuromarketing es Fenestra (2011), quien refiere que "el Neuromarketing es una disciplina moderna, producto de la convergencia de las neurociencias y marketing".

La aplicación de esta herramienta parte de la utilización de técnicas que pertenecen a la neurociencia, utilizadas en el ámbito del marketing. Se consideran características claves como son la atención, emoción y memoria, elementos esenciales para entender la lógica de consumo, comprender los deseos, impulsos y motivaciones de los individuos a través del estudio de las reacciones neurológicas a ciertos estímulos.

Algunos expertos en el tema destacan que los 3 factores anteriormente mencionados son los que más influyen en las decisiones de compra. Algunas investigaciones hechas por neurólogos explican que la mayor parte de todas las decisiones de compra se toman en el nivel subconsciente y que, por lo tanto, es importante tomar en cuenta el tipo de recuerdo para poder resolver problemas de marketing y poder establecer acciones que permitan mejorar y alcanzar los distintos objetivos.

Según Zurawicki (2012), "el Neuromarketing se apoya en tecnologías de diagnósticos para identificar patrones de actividades cerebrales que revelen los mecanismos internos del individuo cuando es expuesto a determinados estímulos" (pág.18)

El propósito de aplicar este tipo de herramienta, es comprender de mejor manera el comportamiento de los consumidores a través de la identificación de los mecanismos cerebrales implicados en la compra por ese motivo, y describir los elementos conscientes y no conscientes de la mente del consumidor, e incluso retroalimentar y generar resultados positivos o negativos por lo que el - Neuromarketing se puede definir como: 
- El estudio a través de las neurociencias, del funcionamiento del cerebro, los seres humanos cuando se exponen a estímulos que pueden ser marcas registradas, productos, olores, o anuncios.

- Mejores herramientas de persuasión.

De acuerdo a lo expuesto anteriormente, el Neuromarketing va enfocado al estudio de estrategias que pueden ayudar a mejorar la imagen, comercialización, rentabilidad, publicidad.

Pueden identificarse seis grandes donde se la utilización del Neuromarketing resulta muy efectiva:

- Marca: Las marcas son ideas en la mente que obtienen fuerzas de las conexiones que se hacen con el Neuromarketing ofreciendo poderosas técnicas para la medición de las asociaciones de marca.

- El diseño del producto y la innovación: En el mismo se pueden medir las respuestas de los consumidores a las ideas de productos y diseños de paquete que son en gran medida automáticas, emocionales y fuera de nuestra percepción consciente como vendedores.

- Efectividad de la publicidad: Los impactos publicitarios son de gran relevancia para los vendedores ya que se llevan a cabo a través de medios no conscientes sabiendo reconocer que no se cree que lo hace, por medio del Neuromarketing se explica detalladamente el cómo se lo realiza.

- Toma de decisiones del comprador: Neuromarketing muestra cómo almacenar los entornos que influyen directamente en los compradores al decidir y comprar, no tratándose de un proceso lógico.

- Experiencias en línea: El mundo en línea ofrece nuevos desafíos a nuestros viejos cerebros. La ciencia del cerebro muestra las diversas maneras en que se puede ser influenciado sutilmente a medida que se avanza en las actividades propias que se ejecutan en línea.

- Entretenimiento eficacia: Entretenimiento crea experiencias en las mentes de las personas que pueden influir en las actitudes, preferencias y acciones. El Neuromarketing muestra lo que ocurre cuando el entretenimiento trasporta al cliente a un mundo imaginario. 
Es fundamental dentro del marketing determinar la comprensión del proceso perceptivo, ya que lo que el cliente percibe, se transforma en la verdadera realidad basado en la visión que tiene del mundo que lo rodea, recordando que se derivarán comportamientos, los cuales son de suma utilidad para las empresas que buscan intereses dentro del mercado y así poder relacionar el campo de la neurociencia con el marketing para adaptar a la realidad del consumidor los productos, bienes o servicios, mensajes, envases, entre otros, a la realidad que perciben los clientes.

En la actualidad se deben realizar tomas de decisiones oportunas en la que se puedan especificar estrategias para mejorar los vínculos entre la empresa y los clientes, así recuperar posicionamiento y fidelización de los clientes, enfocándose directamente al cliente y no solo a los ingresos que tienen las empresas, hay que determinar estrategias que se apliquen para mejorar en el mercado en cuanto a los clientes actuales y potenciales.

Las empresas buscan mejorar sus procesos, gestión, comercialización, ventas, atención al cliente y sobre todo dirigido a mejorar su rentabilidad, por ello utilizan herramientas basadas en Neuromarketing para ayudar a medir con mayor precisión las preferencias en el momento de compra, dependiendo de patrones lógicos que hay que estudiarlos y para ello se puede conseguir beneficios como:

1. Evalúa lo que el consumidor piensa y siente en vez de lo que cuenta.

2. Suprime el factor "adivinar" o "suponer" del trabajo tradicional de estudio de mercado

3. Proporciona datos más profundos que los meramente cuantitativos tradicionales.

4. Mide toda forma de estímulo ante un modelo sin necesidad de preguntar

5. Identifica patrones de respuesta causa efecto.

6. Consigue rendimiento más eficaz y profundo.

7. Aplica los últimos principios y conocimientos de los procesos cognitivos de las neurociencias y la neuropsicología.

8. Aporta los mejores diagnósticos para la optimización de un proyecto. 
9. Alcanza y cuantifica partes de conocimiento que otras técnicas de investigación no pueden: emociones, sentimientos, trabajo intangible, experiencial y sensorial.

10. Aporta entendimiento al pensamiento del consumidor y a su percepción.

El establecer de manera correcta la utilización de esta herramienta dentro del desarrollo del marketing, podría desarrollar ventajas en los sentidos neuronales que posee el cerebro.

Algunas de las ventajas que pueden mencionarse de la aplicación del neuromarketing en el ámbito empresarial son:

- Optimiza las técnicas y recursos publicitarios con el fin de alcanzar la relación entre la mente y la conducta del consumidor, labor que en la actualidad se considera un reto para el marketing.

- Corporativamente el Neuromarketing disminuye el riesgo empresarial, puesto que se crean productos que están sujetos a las necesidades de los consumidores.

- El valor agregado del Neuromarketing es la precisión que puede facilitar en un estudio debido a que sus herramientas son más atinadas en cuanto a que la persona estudiada no puede manipular las respuestas por el inconsciente el que realmente responde a los estímulos.

Sin embargo, a pesar de reconocer lo anterior no puede obviarse que la utilización del neuromarketing como herramienta también tiene algunas implicaciones negativas como son:

- Este método no considera la subjetividad del sujeto, es decir, sus experiencias, ideas, sentimientos, etc. Lo cual no certifica que éste funcione en todos los consumidores.

- Algunos consumidores pueden considerar estas "técnicas", invasivas a su intimidad, puesto que estas orientan sus emociones hacia el mercado. Esto puede incidir en que los clientes pueden llegar a sentirse manipulados al momento de adquirir un producto.

- Realizar un estudio de neuromarketing tiene un costo elevado en contraste con otros métodos similares, ya que emplea herramientas como resonancia magnética, etc. Lo cual limita su uso para todo el sector del marketing. 
En general, hay que tener en cuenta que los estímulos que proceden de una estrategia de Neuromarketing aplicado al Marketing, como producto, marca, precios, canales de comunicación, anexados a todos esos factores como la experiencia, incentivos, sensaciones son transmitidas al cerebro de los individuos donde enlazan el cableado neuronal que se constituye en la base biológica de las decisiones que tomarán cuando deban elegir cómo, qué, dónde y cuándo comprar o consumir un servicio o producto.

\section{Conclusiones}

El neuromarketing, permite obtener información sobre los procesos mentales que no se perciben de manera consciente, los cuales parecen suponer alrededor del $85 \%$ de las decisiones de compra de los clientes, ya que la mayoría de ellas vendrán mediadas por estímulos subconscientes que ninguna técnica de estudio convencional puede medir.

Con la aplicación del neuromarketing, se podrán optimizar las estrategias de marketing tradicional y, así, mejorar las técnicas y recursos publicitarios y ayudar a comprender la relación entre el cerebro y la conducta del consumidor, algo que no puede lograrse de otro modo hoy día y que constituye el desafío más importante para el marketing del siglo XXI.

\section{Referencias bibliográficas}

Amaya, J. (2012). Gerencia: Planeación \& estrategia. Bogotá: Santo Tomás.

Arquero José Daniel et al (2012). Marketing de Clientes ¿Quién se ha llevado a mi cliente?

México: McGraw-Hill Interamericana.

Braidot, N. (2009). Neuromarketing: Neuroeconomía y Negocios. México D.F: Biblioteca

Braidot.

Braidot, N. (2011). Neuromarketing. Barcelona: Gestión 2000.

Braidot, N. (2012). Neuromarketing: Neuroeconomía y Negocios. Barcelona: Esic.

Callejas, M. (2013). Estructura corporativa. México: Pearson. Recuperado el 17 de marzo de 2016, de Almacenes Stefanie. Disponible en: http://www.stefanie.com.ec/html/almacenes.html

Cayuela, M. (2012). Neuromarketing: cerebrando negocios y servicios. Barcelona: UOC. 
Center, M. P. (2012). Instrumentos de análisis del marketing estratégico. Madrid: Díaz de Santos.

Cisneros, A. (2013). Neuromarketing y neuroeconomía. Bogotá: ECOE EDICIONES.

Feenstra, R. (2011). Ética de la publicidad. Retos en la era digital. Madrid: Dykinson.

Ferrell, M. (2012). Estrategia de marketing. México: Cengage Learning.

García, E. (2011). Marketing y plan de negocio de la microempresa. Barcelona: Paraninfo.

Gronroos, C. (20120). Marketing y gestión de servicios. Madrid: Díaz de Santos.

Kotler, P., \& Armstrong, G. (2011). Fundamentos de marketing. México: Pearson Educación.

Misiego, F. (2012). Neuromarketing político 2da edición. Madrid: Editorial Rasche.

Munuera, J. (2012). Estrategias de marketing: un enfoque basado en el proceso de dirección. Barcelona: ESIC.

Palomo, M. (2014). Atención al cliente. España: Paraninfo, S.A.

Parmelee, D. (2012). Desarrollo exitoso de las estrategias de marketing. Catalunya: Reverte.

Pride, W., \& O, F. (2014). Marketing. California: Cengage.

Psinergia. http://www.centropsinergia.wordpress.com (citado el 12 de marzo de 2016).

Disponible en http://www.centropsinergia.wordpress-psicologia-del-marketing.com

Publishing, M. (2013). La estrategia básica de marketing. Madrid: Díaz de Santos.

Talaya, Á. (2012). Principios de marketing. Madrid: Esic.

Vilma Núñez. (Recuperado el 26 de agosto de 2016). Disponible en: http://www.vilmanunezmarketing-de-influencia.com

Viudes, P. (2011). Gestión Estratégica. Medellín: De Santo. (Recuperado el 13 de Agosto de 2016). Disponible en: http://www.presenceco.com

Zurawicki, L. (2012). Neuromarketing: Exploring the Brain of the Consumer. Barcelona: IICA. 